\title{
Effect of social support on depression of internet addicts and the mediating role of loneliness
}

Fei He ${ }^{*}$, Qin Zhou ${ }^{2}$, Jing Li ${ }^{1}$, Rong Cao ${ }^{1}$ and Hao Guan ${ }^{2 *}$

\begin{abstract}
Background: Many studies have determined the existence of an extremely close association between Internet addiction and depression. However, the reasons for the depression of Internet addicts have not been fully investigated.

Aim: This cross-sectional study aims to explore the factors that influence depression among Internet addicts.

Methods: A total of 162 male Internet addicts completed the Emotional and Social Loneliness Scale, Multidimensional Scale of Perceived Social Support, and Self-Rating Depression Scale.

Results: Loneliness and lack of social support are significantly correlated with depression among Internet addicts. Structural Equation Modeling results indicate that social support partially mediates loneliness and depression.

Conclusions: Both social support and loneliness were negatively associated with depression of Internet addicts whereas loneliness plays a mediating role between social support and depression.
\end{abstract}

Keywords: Internet addiction, Depression, Social support, Loneliness, Structural equation modeling

\section{Background}

Since the late 1990s, the Internet has entered a rapid development track in China [1]. On one hand, the Internet has allowed adolescents to enjoy the unprecedented convenience and pleasure brought about by modern technological civilization in the virtual world of the Internet, particularly in learning and communicating [2]. On the other hand, the Internet is a "double-edged sword" because of its negative effects on the multifaceted psychological development of the adolescents. Most interesting of these negative effect is Internet addiction [3-5]. Internet addiction, also known as pathological Internet use (PIU), is a multi-dimensional syndrome comprising cognitive and behavioral symptoms, which generally leads to social withdrawal, truancy, loneliness, and depression [6-11].

Studies have found a very close association between Internet addiction and depression [12-14]. Young reported that level of depression is highly correlated with Internet addiction [15]. Other researchers found that Internet use and addiction increase the risk of depression among adults and is related with depression and

\footnotetext{
* Correspondence: hefei@nwu.edu.cn; guanhaofmmu@126.com

'School of Public Management, Northwest University, Xi'an, China

${ }^{2}$ Department of Burns and Cutaneous Surgery, Xijing Hospital, Xi'an, China
}

suicidal ideation among adolescents [16,17]. The depression scores of Internet addicts were found to be significantly higher than those of non-addicts [18]. Researchers believe that this depression is due to the addiction of adolescents to the virtual world, which impedes their relationships in the real world. In effect, they lack face-to-face communication and real social support, which increases their susceptibility to depression $[19,20]$.

Loneliness is a painful subjective experience or psychological feeling, which is characterized by the lack of satisfactory relationships [21]. Sullivan believes that loneliness is an unpleasant experience arising from unfulfilled intimate or close interpersonal needs [22]. Michela et al. [23] view loneliness as the unpleasant experience of individuals brought about by the realization that a gap exists between the expected and actual results in relation to the social network model. Russell, Peplau, and Ferguson believe that loneliness is a subjective social isolation state stemming from a person's painful experience of not being accepted by others [24]. The relationship among social support, depression, and loneliness among adolescents has been defined by various studies. Many studies have focused on the effects of social support from a specific source, and showed a significant negative correlation between the 
support from parents, companions, or teachers and the depression and loneliness of adolescents [19,25-28].

Previous findings have revealed that Internet addicts are prone to depression, and depression is associated with loneliness and the lack of social support. However, these findings failed to combine the two issues: (1) Can social support and loneliness affect depression of Internet addicts? (2) What is the trilateral relationship among social support, loneliness and depression? We hypothesized that both social support and loneliness have significant impacts on the depression of Internet addicts, and that loneliness plays a mediating role between social support and depression.

\section{Methods}

\section{Participants}

The participants consisted of 1,024 male undergraduate students, with ages between 19 and 23, from four Xi'an universities, which have comprehensive course offerings. A total of 1,024 questionnaires were distributed, and 1,023 were collected. Thirty-three questionnaires were partially filled out and thus, were discarded. A total of 990 questionnaires were considered valid. The Internet addiction test employed the clinical diagnosis questionnaire designed by Young. The revised Internet addiction test comprises eight items answerable by yes or no. The subject was regarded as an Internet addict if five or more of his answers were positive [29]. Of the 990 subjects, $162(16.36 \%)$ were Internet addicts. Subsequently, these participants were asked to take more measures.

\section{Instruments}

\section{Multidimensional scale of perceived social support}

The Multidimensional Scale of Perceived Social Support is a 12-item instrument with a seven-point scale $(1=$ strongly disagree; $7=$ strongly agree) that measures three sources of support, namely, family, friends, and significant others [30]. Items in this scale include "My family really tries to help me", "My friends really try to help me", and "There is a special person in my life who cares about my feelings". The scale was translated into Chinese by Chou [31], and has been proved to have good validity and reliability in Chinese populations. In the present study, the Cronbach alpha coefficient for Multidimensional Scale of Perceived Social Support was 0.808.

\section{Social and emotional loneliness scale}

The Social and Emotional Loneliness Scale was developed by Wittenberg, which consists of 10 items designed to assess social loneliness (SL) and emotional loneliness (EL). Responses to each item are given on a five-point Likert-type scale ranging from 1 (never) to 5 (very often). It includes items such as, "1 have a really nice set of friends"; "I have friends and acquaintances with who I like to be together". Scale scores are the sum of items with reverse coding of relevant items [32]. The Social and Emotional Loneliness Scale was translated into Chinese by Kong and You [33]. In the current study, the Cronbach alpha coefficient for the Social and Emotional Loneliness Scale was 0.787 .

\section{Self-rating depression scale (SDS)}

Self-rating Depression Scale (SDS), developed by Zung, is a self-report measure of depression consisting of 20 items, with a four-point scale ranging from a little of the time (1) to most of the time (4). Of the 20 items, 10 are worded positively and 10 are worded negatively. The former 10 items are reversed items. The validity and the reliability of the SDS have been reported [34]. The researcher translated the 20-item version of the Self-rating Depression Scale into Chinese, and the Chinese vision of the scale has been proved to have good validity and reliability [35]. In the current study, the Cronbach alpha coefficient for SDS was 0.851 .

\section{Data analysis}

Firstly, the intercorrelations of all the latent variables were calculated to test the significant of correlation coefficient. Then, a two-step procedure introduced by Anderson and Gerbing was adapted to analyses the mediation effect in order to confirm the structural relations of the latent structured model [36]. Firstly, the measurement model of the four latent variables was tested to assess the extent of goodness of fit represented by its indicators respectively [37]. If index of confirmatory measurement model meet the requirements, then the maximum likelihood estimation would be used to test the SEM [38]. All the above analysis was conducted in AMOS 17.0 program.

The following four indices were utilized to evaluate the goodness of fit of the model: (a) Chi-square statistic $\left(x^{2}\right), x^{2} / d f,(b)$ the Standardized Root Mean Square Residual (SRMR), (c) the Root Mean Square Error of Approximation (RMSEA), and (d) the Comparative Fit Index (CFI) [39-41]. In this study, a model was considered to have a good fit if all the path coefficients were significant at the level of $0.05, \mathrm{X}^{2} / \mathrm{df}$ was below 5, SRMR was below 0.08, RMSEA was below 0.08, and CFI was 0.95 or more.

\section{Results}

\section{Descriptive statistics and correlation analysis}

Means, standard deviations, and intercorrelations of all variables are presented in Table 1 . The results indicate that social support is negatively correlated with loneliness $(\mathrm{r}=-0.44, \mathrm{p}<0.01)$ and depression $(\mathrm{r}=-0.42, \mathrm{p}<$ 0.01 ). By contrast, loneliness is positively correlated with depression $(\mathrm{r}=0.33, \mathrm{p}<0.01)$. 
Table 1 Means, standard deviations, and correlations of the variables of interest

\begin{tabular}{llllll}
\hline & Mean & SD & $\mathbf{1}$ & $\mathbf{2}$ & $\mathbf{3}$ \\
\hline 1. Social support & 66.81 & 12.17 & 1 & & \\
2. Loneliness & 32.15 & 6.42 & $-0.44^{* *}$ & 1 & \\
3. Depression & 66.03 & 8.08 & $-0.42^{* *}$ & $0.33^{* *}$ & 1 \\
\hline
\end{tabular}

Note: ${ }^{* *} \mathrm{p}<0.01$.

\section{Measurement model}

Confirmatory factor analysis (CFA) was adopted to assess whether or not the measurement model adequately fits the sample data. The initial test of the measurement model revealed a satisfactory fit $\left[X^{2}(\mathrm{df}=17)=21.50, \mathrm{X}^{2} /\right.$ $\mathrm{df}=1.27 ; \quad \mathrm{RMSEA}=0.041 ; \quad \mathrm{SRMR}=0.046$ and $\mathrm{CFI}=$ 0.993]. All the factor loadings for the indicators on the latent variables were significant $(\mathrm{p}<0.001)$, indicating that all the latent constructs are well-represented by their indicators.

\section{Structural model}

To analyze the mediation effect, structural equation modeling (SEM) was used. First, the direct effect of social support on depression without mediators was tested. The directly standardized path $(\beta=-0.47, \mathrm{p}<0.001)$ was significant. After which, a partially-mediated model containing a mediator (loneliness) and a direct path from social support to depression was tested. All the path coefficients were statistically significant, see Figure 1: The results showed an acceptable fit of the model to the data $\left[\chi^{2}\right.$ $(\mathrm{df}=17)=21.50, \quad \chi^{2} / \mathrm{df}=1.27 ; \quad \mathrm{RMSEA}=0.041 ; \quad$ SRMR $=$ 0.046 and CFI $=0.993$ ]. These results showed that social support and loneliness have significant effects on depression among Internet addicts.

\section{Confidence interval of direct and indirect effects}

The mediating effects of loneliness on social support and depression were tested for significance by adopting the
Bootstrap estimation procedure in AMOS (a bootstrap sample of 1,000 was specified). Sobel test, the commonly employed method for examining statistical significance of a mediation effect, which involves computing the ratio of products of direct effects to their estimated standard error, was not considered. The test requires the products of direct effects to follow a normal distribution, which does not generally occur, resulting in the reduction of statistical efficacy. By contrast, the bootstrap test relies on 95\% confidence intervals from the empirical distribution of indirect effect estimates. According to Mackinnon [40,42,43], the bootstrap method yields the most accurate confidence intervals for indirect effects. Table 2 shows the indirect effects and their associated 95\% confidence intervals. The indirect effect of social support on depression through loneliness is significant.

\section{Discussion}

The current study examines the effect of loneliness and social support on depression among Internet addicts. As expected, the correlational findings of the study indicate that social support has a negative relationship with depression and loneliness. The result of SEM indicates that loneliness partially mediates the relationship between social support and depression.

Furthermore, social support has a significant predication effect on depression, which is consistent with the previous conclusion that states that social support is a kind of available resource, which allows Internet addicts to re-evaluate the produced negative emotions that directly reduce their depression and anxiety to some extent [44]. In addition, a good social support can reduce the feeling of pressure, which motivates an individual to improve one's perceived self-response capacity toward alleviating the evaluation of the severity of stressful events, and reducing the adverse effects of the stressful experience [45]. Social support is significantly associated with loneliness: the lesser the feeling of social support, the

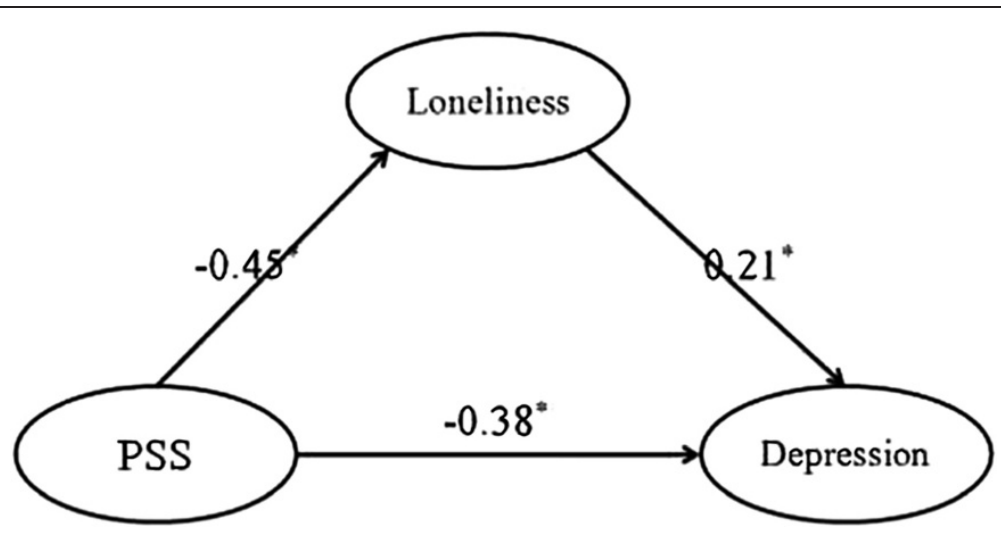

Figure 1 The impact of social support and loneliness on depression of Internet addicts. Note: Factor loadings are standardized ( $p<0.05$ ). 
Table 2 Direct and indirect effects and $95 \%$ confidence intervals for the final model

\begin{tabular}{llll}
\hline Model pathways & $\begin{array}{l}\text { Estimated } \\
\text { effect }\end{array}$ & $\begin{array}{l}\mathbf{9 5 \%} \mathrm{Cl} \\
\text { Lower } \\
\text { bonds }\end{array}$ & $\begin{array}{l}\mathbf{9 5 \%} \mathrm{Cl} \\
\text { Up } \\
\text { bonds }\end{array}$ \\
\hline Direct effect & & & \\
Social support $\rightarrow$ Depression & -0.378 & -0.192 & -0.551 \\
Social support $\rightarrow$ Loneliness & -0.455 & -0.209 & -0.659 \\
Loneliness $\rightarrow$ Depression & 0.211 & 0.035 & 0.430 \\
$\begin{array}{l}\text { Indirect effect } \\
\text { Social support } \rightarrow \text { Loneliness } \rightarrow\end{array}$ & -0.096 & -0.012 & -0.246 \\
Depression & & & \\
\hline
\end{tabular}

higher the feeling of loneliness [46,47]. Loneliness is essentially a state where an individual feels the lack of satisfactory relationships and a subjective psychological feeling or experience where a gap arises between the personal desire for interaction and the actual level of interaction [48]. The current study found that loneliness can partially mediate the effect of social support on the depression of Internet addicts. Loneliness is a closed and diffused mental state, and a depressed mood arising from the feeling of isolation and exclusion; and the individual's perception of the quality and quantity of his own social interaction, often accompanied by loneliness and helplessness, as well as other negative emotions and spiritual emptiness, which consequently produces depression $[21,49]$.

The study concludes that the Internet causes addiction, which in turn leads to the lack of social support. The lack of social support, on one hand, can directly lead to depression; on the other hand, it can indirectly affect depression through the mediation of loneliness. In summary, this study has provided insights into the relationship among social support, loneliness, and depression. In particular, social support acts as a protective factor by decreasing the feelings of loneliness, which in turn creates a beneficial effect on depression. Thus, to enhance the mental health level of Internet addicts, adequate social support should be provided to address the feelings of isolation and exclusion. Despite these achievements, the present study has limitations. First, the study's correlational cross-sectional nature prohibits the drawing of any causal relationships among the variables. Thus, caution must be exercised when interpreting the results of meditational analyses on cross-sectional data. Future longitudinal or experimental studies should be conducted to facilitate more causal evaluations. Second, loneliness was the only potential mediator that was examined despite the presence of other mediators that capable of playing an important role in the relationship between social support and depression. Thus, other variables, such as self esteem, should be examined. Third, the data in this study were collected only through self-report scales. The use of multiple methods for evaluation may have decreased the "subjectivity" limitation of the findings.

\section{Competing interests}

The authors declare that they have no competing interests.

\section{Authors' contributions}

Conceived and designed the experiments: FH, HG. Performed the experiments: FH, QZ, JL, RC, HG. Analyzed the data: JZ, JP. Contributed reagents/materials/analysis tools: $\mathrm{FH}, \mathrm{HG}$. Wrote the paper: FH, HG. All authors read and approved the final manuscript.

\section{Acknowledgement}

This study was supported by Social Science foundation of the People's Republic of China (13XRK004), Natural Science Grant of Shann'xi Province (2012K18-03-06), and Social Science fund of China (13BGL074).

Received: 22 June 2014 Accepted: 12 August 2014

Published: 16 August 2014

\section{References}

1. Srikantaiah TK, Xiaoying D: The Internet and its impact on developing countries: examples from China and India. Asian Libr 1998, 7(9):199-209.

2. Javalgi RRG, Radulovich LP, Pendleton G, Scherer RF: Sustainable competitive advantage of internet firms: a strategic framework and implications for global marketers. Int Mark Rev 2005, 22(6):658-672.

3. Ko CH, Liu GC, Yen JY, Chen CY, Yen CF, Chen CS: Brain correlates of craving for online gaming under cue exposure in subjects with Internet gaming addiction and in remitted subjects. Addict Biol 2013, 18(3):559-569.

4. Winkler A, Dörsing B, Rief W, Shen Y, Glombiewski JA: Treatment of Internet addiction: a meta-analysis. Clin Psychol Rev 2013, 33(2):317-329.

5. Kraut JA, Madias NE: Association of serum bicarbonate with clinical outcomes in CKD: could an increase in serum bicarbonate be a double-edged sword? Am J Kidney Dis 2013, 62(4):647-649.

6. Davis RA: A cognitive-behavioral model of pathological internet use. Comput Hum Behav 2001, 17(2):187-195.

7. Morahan-Martin J, Schumacher P: Incidence and correlates of pathological internet use among college students. Comput Hum Behav 2000, 16(1):13-29.

8. Spada MM: An overview of problematic internet use. Addict Behav 2014, 39(1):3-6.

9. Starcevic V: Is Internet addiction a useful concept? Aust N Z J Psychiatry 2013, 47(1):16-19.

10. Lee YS, Han DH, Kim SM, Renshaw PF: Substance abuse precedes internet addiction. Addict Behav 2013, 38(4):2022-2025.

11. Wallace P: Internet addiction disorder and youth. EMBO Rep 2014, 15(1):12-16.

12. Yang $L$, Sun $L$, Zhang $Z$, Sun $Y, W u H$, Ye D: Internet addiction, adolescent depression, and the mediating role of life events: finding from a sample of Chinese adolescents. Int J Psychol 2014, doi:10.1002/ijop.12063.

13. Kim K, Ryu E, Chon M-Y, Yeun E-J, Choi S-Y, Seo J-S, Nam B-W: Internet addiction in Korean adolescents and its relation to depression and suicidal ideation: a questionnaire survey. Int J Nurs Stud 2006, 43(2):185-192.

14. Lin I, Ko C-H, Chang Y-P, Liu T-L, Wang P-W, Lin H-C, Huang M-F, Yeh Y-C, Chou W-J, Yen C-F: The association between suicidality and internet addiction and activities in Taiwanese adolescents. Compr Psychiatry 2014, 55(3):504-510.

15. Young KS, Rogers RC: The relationship between depression and internet addiction. Cyberpsychol Behav 1998, 1(1):25-28.

16. Byun S, Ruffini C, Mills JE, Douglas AC, Niang M, Stepchenkova S, Lee SK Loutfi J, Lee J-K, Atallah M: Internet addiction: metasynthesis of 19962006 quantitative research. Cyberpsychol Behav 2009, 12(2):203-207.

17. Yen J-Y, Ko C-H, Yen C-F, Wu H-Y, Yang M-J: The comorbid psychiatric symptoms of internet addiction: attention deficit and hyperactivity disorder (ADHD), depression, social phobia, and hostility. J Adolesc Health 2007, 41(1):93-98.

18. Yang SC, Tung C-J: Comparison of Internet addicts and non-addicts in Taiwanese high school. Comput Hum Behav 2007, 23(1):79-96.

19. Shaw $L H$, Gant $L M$ : In defense of the internet: the relationship between Internet communication and depression, loneliness, self-esteem, and perceived social support. Cyberpsychol Behav 2002, 5(2):157-171. 
20. Sanders CE, Field TM, Diego M, Kaplan M: The relationship of Internet use to depression and social isolation among adolescents. Adolescence 2000, 35(138):237-242

21. Liu L, Gou Z, Zuo J: Social support mediates loneliness and depression in elderly people. J Health Psychol 2014, doi:10.1177/1359105314536941.

22. Sønderby LC, Wagoner B: Loneliness: an integrative approach. J Integr Soc Sci 2013, 3(1):1-29.

23. Michela $J$, Peplau LA, Weeks DG: Perceived dimensions of attributions for loneliness. J Pers Soc Psychol 1982, 43(5):929-936.

24. Russell D, Peplau LA, Ferguson ML: Developing a measure of loneliness. J Pers Assess 1978, 42(3):290-294.

25. Hudson DB, Elek SM, Campbell-Grossman C: Depression, self-esteem, loneliness, and social support among adolescent mothers participating in the new parents project. Adolescence 2000, 35(139):445-453.

26. Adam EK, Chyu L, Hoyt LT, Doane LD, Boisjoly J, Duncan GJ, Chase-Lansdale PL, McDade TW: Adverse adolescent relationship histories and young adult health: cumulative effects of loneliness, low parental support, relationship instability, intimate partner violence, and loss. J Adolesc Health 2011, 49(3):278-286.

27. Kleinberg A, Aluoja A, Vasar V: Social support in depression: structural and functional factors, perceived control and help-seeking. Epidemiol Psychiatr Sci 2013, 22(04):345-353.

28. Chen Y, Hicks A, While AE: Loneliness and social support of older people in China: a systematic literature review. Health Soc Care Comm 2014, 22(2):113-123.

29. Young KS: Psychology of computer use: XL. Addictive use of the Internet: a case that breaks the stereotype. Psychol Rep 1996, 79(3):899-902.

30. Zimet GD, Dahlem NW, Zimet SG, Farley GK: The multidimensional scale of perceived social support. J Pers Assess 1988, 52(1):30-41.

31. Chou K-L: Assessing Chinese adolescents' social support: the multidimensional scale of perceived social support. Personal Individ Differ 2000, 28(2):299-307.

32. Shaver PR, Brennan KA: Measures of depression and loneliness. Meas Pers Soc Psychol Attitudes 1991, 1:195-290.

33. Kong F, You $X$ : Loneliness and self-esteem as mediators between social support and life satisfaction in late adolescence. Soc Indic Res 2013, 110(1):271-279.

34. Zung W: Zung self-rating depression scale and depression status inventory. In Assessment of Depression. Edited by Sartorius N, Ban T. Berlin: Springer; 221-231.

35. Lee HC, Chiu HFK, Wing YK, Leung CM, Kwong PK, Chung DWS: The Zung self-rating depression scale: screening for depression among the Hong Kong Chinese elderly. J Geriatr Psychiatry Neurol 1994, 7(4):216-220.

36. Anderson JC, Gerbing DW: Structural equation modeling in practice: a review and recommended two-step approach. Psychol Bull 1988, 103(3):411-423.

37. Xiao W, Zhou L, Wu Q, Zhang Y, Miao D, Zhang J, Peng J: Effects of person-vocation fit and core self-evaluation on career commitment of medical university students: the mediator roles of anxiety and career satisfaction. Int J Ment Heal Syst 2014, 8(1):8.

38. Peng J, Jiang X, Zhang J, Xiao R, Song Y, Feng X, Zhang Y, Miao D: The impact of psychological capital on job burnout of Chinese nurses: the mediator role of organizational commitment. PLOS ONE 2013, 8(12):e84193.

39. Lt H, Bentler PM: Cutoff criteria for fit indexes in covariance structure analysis: conventional criteria versus new alternatives. Struct Equ Model 1999, 6(1):1-55.

40. He F, Cao R, Feng Z, Guan H, Peng J: The impacts of dispositional optimism and psychological resilience on the subjective well-being of burn patients: a structural equation modelling analysis. PLOS ONE 2013, 8(12):e82939.

41. Peng J, Li D, Zhang Z, Tian Y, Miao D, Xiao W, Zhang J: How can core self-evaluations influence job burnout? The key roles of organizational commitment and job satisfaction. J Health Psychol 2014, doi:10.1177/ 1359105314521478.

42. MacKinnon DP, Lockwood CM, Hoffman JM, West SG, Sheets V: A comparison of methods to test mediation and other intervening variable effects. Psychol Methods 2002, 7(1):83.

43. Zhang J, Wu Q, Miao D, Yan X, Peng J: The impact of core self-evaluations on job satisfaction: the mediator role of career commitment. Soc Indic Res 2014, 116(3):809-822.
44. Park J, Kitayama S, Karasawa M, Curhan K, Markus HR, Kawakami N, Miyamoto Y, Love GD, Coe CL, Ryff CD: Clarifying the links between social support and health: culture, stress, and neuroticism matter. J Health Psychol 2013, 18(2):226-235.

45. Coulson NS: Receiving social support online: an analysis of a computermediated support group for individuals living with irritable bowel syndrome. Cyberpsychol Behav 2005, 8(6):580-584.

46. Golden J, Conroy RM, Bruce I, Denihan A, Greene E, Kirby M, Lawlor BA: Loneliness, social support networks, mood and wellbeing in communitydwelling elderly. Int J Geriatr Psychiatr 2009, 24(7):694-700.

47. Tomaka J, Thompson S, Palacios R: The relation of social isolation, loneliness, and social support to disease outcomes among the elderly. J Aging Health 2006, 18(3):359-384.

48. Steptoe A, Shankar A, Demakakos P, Wardle J: Social isolation, loneliness, and all-cause mortality in older men and women. Proc Natl Acad Sci 2013, 110(15):5797-5801.

49. Jaremka LM, Fagundes CP, Glaser R, Bennett JM, Malarkey WB, Kiecolt-Glaser JK Loneliness predicts pain, depression, and fatigue: understanding the role of immune dysregulation. Psychoneuroendocrinology 2013, 38(8):1310-1317.

doi:10.1186/1752-4458-8-34

Cite this article as: He et al:: Effect of social support on depression of internet addicts and the mediating role of loneliness. International Journal of Mental Health Systems 2014 8:34.

\section{Submit your next manuscript to BioMed Central and take full advantage of:}

- Convenient online submission

- Thorough peer review

- No space constraints or color figure charges

- Immediate publication on acceptance

- Inclusion in PubMed, CAS, Scopus and Google Scholar

- Research which is freely available for redistribution

Submit your manuscript at www.biomedcentral.com/submit
C) Biomed Central 